\title{
The clinical safety and efficacy of flexible bronchoscopy in a neonatal intensive care unit
}

\author{
CHONGBING YAN, YONG HU, GANG QIU, XIAOHUI GONG and DERMYSHI ELDA
}

Department of Neonatology, Shanghai Children's Hospital, Shanghai 200062, P.R. China

Received April 6, 2020; Accepted July 22, 2020

DOI: $10.3892 /$ etm.2020.9223

\begin{abstract}
Flexible bronchoscopy (FB), developed in the 1960 s, is widely used in the clinical practice of pediatrics and has demonstrated fundamental value in clinical diagnoses and treatment. However, as an invasive procedure, the use of FB is limited due to concerns regarding the tolerance of the procedure and the possible complications in neonatal units. Thus, the present study aimed to investigate the clinical safety and efficacy of flexible bronchoscopy (FB) in a neonatal intensive care unit (NICU). Neonates $(n=54)$ who received FB in the NICU of Shanghai Children's Hospital between January 2012 and December 2016 were enrolled as the experimental group and another 54 neonates who required nebulization and tracheal secretion suction treatments were the control group. Indicators including blood gas, complete blood count, C-reactive protein (CRP), X-ray, patient breathing rate, temperature and blood pressure were monitored prior to and following the procedure. No significant differences in sex, gestational age, birth weight or postnatal age were observed between the experimental group and the control group $(\mathrm{P}>0.05)$. Among the $54 \mathrm{FB}$ patients, several cases with side effect were identified, including 18 (33.3\%) with respiratory tract stenosis, nine $(16.7 \%)$ with malacia and stenosis and six (11.1\%) with esophagotracheal fistula. Among the 54 members of the control group, 44 neonates $(81.4 \%)$ were discharged with improved condition, five $(9.3 \%)$ succumbed and five patients $(9.3 \%)$ abandoned the treatment and left the hospital. Bronchoalveolar lavage demonstrated consistent results with respiratory secretion culture or tracheal tube culture. In comparison between the experimental and the control groups, no significant difference in $\mathrm{pH}$, partial pressure of carbon dioxide $\left(\mathrm{PCO}_{2}\right)$, partial pressure of oxygen $\left(\mathrm{PO}_{2}\right)$ and $\mathrm{HCO}_{3}{ }^{-}$was observed, while there were no statistical differences in the values of $\mathrm{pH}, \mathrm{PCO}_{2}$ and
\end{abstract}

Correspondence to: Dr Yong Hu, Department of Neonatology, Shanghai Children's Hospital, 355 Luding Road, Shanghai 200062, P.R. China

E-mail: yonghu33x@163.com

Key words: flexible bronchoscopy, neonatal intensive care unit, tracheomalacia, bronchoalveolar lavage, esophagotracheal fistula
$\mathrm{HCO}_{3}{ }^{-}(\mathrm{P}>0.05)$. However, $\mathrm{PO}_{2}$ was significantly increased, and CRP was significantly reduced, following $\mathrm{FB}$ procedure compared with prior to FB $(\mathrm{P}<0.05)$. No pneumothorax, shock, other severe complications, fever or diffused pneumonia were observed during or after FB. The data from the present study demonstrated that FB is a safe and effective strategy for the diagnosis and differentiation of neonatal respiratory diseases in NICU.

\section{Introduction}

Flexible bronchoscopy (FB) was developed in the 1960s. The principle mechanism is that light, originating from an external source, is transmitted into the airway by optical waveguide fiber $(1,2)$. The endoscopic field can be observed by eye, or in the case of modern bronchoscopic devices, on a monitor. The condition of trachea and bronchia can be clearly observed and determined by observation. FB functions as an important diagnostic tool to identify the etiology of respiratory diseases (3). Some abnormalities, such as tracheal-malacia or stenosis, which cannot be diagnosed by radiological images, can be confirmed by FB (4). It can also be used to move foreign bodies in the respiratory tract. Currently, FB is widely used in the clinical practice of pediatrics (Table I). It has demonstrated fundamental value in clinical diagnoses and treatment $(5,6)$. However, as an invasive procedure, the use of FB is limited due to concerns regarding the tolerance of the procedure and the possible complications in neonatal units. The purpose of the present study was to evaluate the safety and efficacy of FB in the neonatal intensive care unit (NICU).

\section{Subjects and methods}

Neonates and criteria. A total of 54 FB neonates admitted to the NICU of Shanghai Children's Hospital (Shanghai, China) between January 2012 and December 2016 were enrolled in the present study according to the Pediatric Bronchoscopy Guidelines (6). Among the 54 FB neonates in experimental group, $37(68.5 \%)$ were male and 17 (31.5\%) were female. Gestational age ranged from 207 to 290 days (261.87 \pm 19.72 days). The birth weight ranged from 1,300 to $4,400 \mathrm{~g}(2,889.63 \pm 675.55 \mathrm{~g})$. The postnatal age ranged from 2 to 290 days $(29.2 \pm 27.79$ days). Inclusion criteria were neonates with recurrent dyspnea or suspicious 
respiratory tract anomaly; with recurrent pulmonary infection or atelectasis in the same lung lobe; with suspicious tracheal stenosis in radiological image [X-ray or computed tomography (CT) scan]; inability to be extubated without clear reason; or confirmed congenital esophageal atresia to clarify the presence and position of esophagobronchial fistula before surgery (Fig. 1). Exclusion criteria were multiple organ dysfunction; severe respiratory diseases with high values of ventilation; severe congenital heart disease or cardiac function failure; coagulopathy; present hyperthermia; or preterm weight $<1,500 \mathrm{~g}$. Data were recorded in detail, including gestational age, postnatal age, birth weight, corrected gestational age while receiving $\mathrm{FB}$, hospital stays, ventilation status and outcomes. Vital signs reflecting FB procedure, such as breathing rate, heart rate and oxygen saturation $\left(\mathrm{SaO}_{2}\right)$, were monitored. Bronchoalveolar lavage (BAL) was performed when signs of endobronchitis were identified, and BAL fluid was sampled for laboratory tests including cell count, biochemistry and culture. Indicators including blood gas, CBC, CRP and X-ray at $1 \mathrm{~h}$ before and $1 \mathrm{~h}$ after FB were ascertained. Patient breathing rate, temperature and blood pressure following FB were recorded. Another 54 neonates who required nebulization treatment and tracheal secretion suction were set as the control group. The neonates were also admitted to the NICU of Shanghai Children's Hospital (Shanghai, China) between January 2012 and December 2016. Among the 54 FB neonates, 36 (66.7\%) were male and $18(33.3 \%)$ were female. The birth weight ranged from 1,250 to $4,500 \mathrm{~g}(2,921.26 \pm 743.96 \mathrm{~g})$. The postnatal age ranged from 4 to 282 days $(31.45 \pm 25.67$ days). The present study was reviewed and approved by the Ethics Committee of Shanghai Children's Hospital, China (approval no. 2011-231). All families who participated in the present study voluntarily signed informed consent. The primary diseases diagnosed in the control group were pneumonia, neonatal respiratory distress syndrome and wet lung.

The Olympus BFXP40 bronchoscope (Olympus Corp.) was used for FB. The outer diameter of the probe was $2.8 \mathrm{~mm}$, with a $1.2 \mathrm{~mm}$ operation tunnel. In addition to using the probe to observe the trachea and bronchia, the operation tunnel, through which oxygen suction can be conducted, was employed for BAL. Patients did not eat from $4 \mathrm{~h}$ before FB to prevent vomiting and aspiration during the procedure. In case of complications, such as laryngeal edema, laryngospasm, or pneumothorax, the FB procedure was performed in the resuscitation unit of the NICU. In addition to a bedside oxygen inhalation tube, a tracheal tube, a resuscitation mask and a suction machine were made ready. Midazolam was used as sedation, with $0.1-0.3 \mathrm{mg} / \mathrm{kg}$ intravenous injection adopted prior to the FB procedure. During the FB, midazolam $0.1 \mathrm{mg} / \mathrm{kg} / \mathrm{h}$ intravenous infusion was administered, and lidocaine hydrochloride mucilage was administered as local anesthesia. The patients received oxygen by nasal tube during the procedure.

Statistical analysis. All the statistical analyses were performed using SPSS 17.0 software (SPSS, Inc.). Unpaired Student's t-test was used for the comparison between twogroups and $\chi^{2}$ test was used for enumeration data. $\mathrm{P}<0.05$ was considered to indicate a statistically significant difference.
Table I. Applications of flexible bronchoscopy.

1 Tracheal, bronchial, pulmonary dysplasia or abnormity. Primary alterations include laryngomalacia, tracheobronchomalacia, laryngeal stenosis, tracheobronchial stenosis and esophagotracheal fistula.

Secondary alterations include tracheobronchial compression caused by subglottic hemangioma, pulmonary artery sling, vascular ring, cardiac dilatation, and stenosis caused by intubation.

2 Atelectasis; using bronchoscopy to check and/or offer bronchoalveolar lavage

3 Hemoptysis or blood-stained sputum, using bronchoscopy to investigate the pathogen and perform pathological examination

4 Chronic cough and recurrent respiratory infection

5 Recurrent or persistent stridor or wheezing

6 Diffused or focal lesions in the lungs

7 Pulmonary tuberculosis

8 Removal of foreign bodies

9 Assistance in diagnosis in some thoracic surgery

10 Assistance in difficult intubations

11 Other treatments including cryotherapy, interventional therapy and balloon dilatation therapy

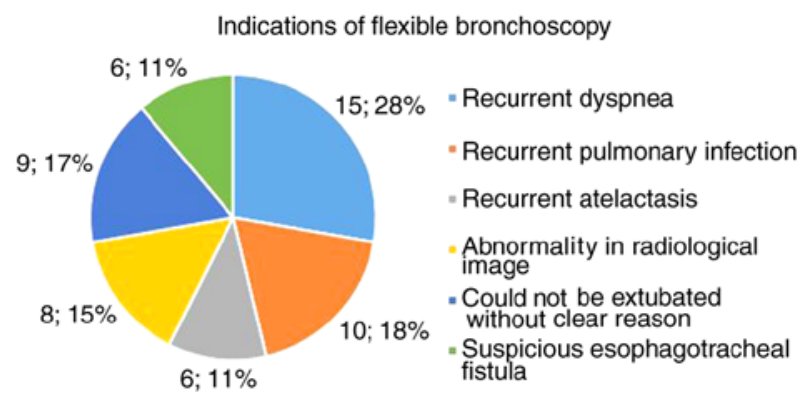

Figure 1. Indications for bronchoscopy. Recurrent dyspnea, 15 cases; Recurrent pulmonary infection, 10 cases; Recurrent atelectasis, 6 cases; Abnormality in radiological image, 8 cases; Could not be extubated without clear reason, 9 cases; Suspicious esophagotracheal fistula, 6 cases.

\section{Results}

General data. A total of $56 \mathrm{FB}$ procedures were conducted with 54 neonates, among whom two received FB twice. Among the 54 neonates, $33(61.1 \%)$ used ventilation and 12 (22.2\%) used nasal continuous positive airway pressure; 8 cases received the FB procedure with intubation. No significant differences in sex, gestational age, birth weight or postnatal age were observed between the experimental and the control groups (P>0.05; Table II).

Results of FB. Among the 54 FB patients, 44 (81.5\%) were identified with varying degrees of airway abnormality. Endobronchitis was observed in 9 cases $(16.7 \%)$, while there was only 1 case $(1.8 \%)$ without any abnormalities. During the 
Table II. General data.

\begin{tabular}{lcccr}
\hline Characteristics & Experimental group $(\mathrm{n}=54)$ & Control group $(\mathrm{n}=54)$ & $\chi^{2}$ or t-test & P-value \\
\hline Sex (male/female) & $37 / 17$ & $34 / 20$ & 0.37 & 0.54 \\
Gestational age (days) & $261.87 \pm 19.72$ & $263.56 \pm 18.31$ & 0.46 & 0.65 \\
Birth weight (g) & $2889.63 \pm 675.55$ & $2915.72 \pm 688.31$ & 0.2 & 0.84 \\
Postnatal age (days) & $29.2 \pm 27.79$ & $28.36 \pm 27.45$ & 0.16 & 0.87 \\
\hline
\end{tabular}

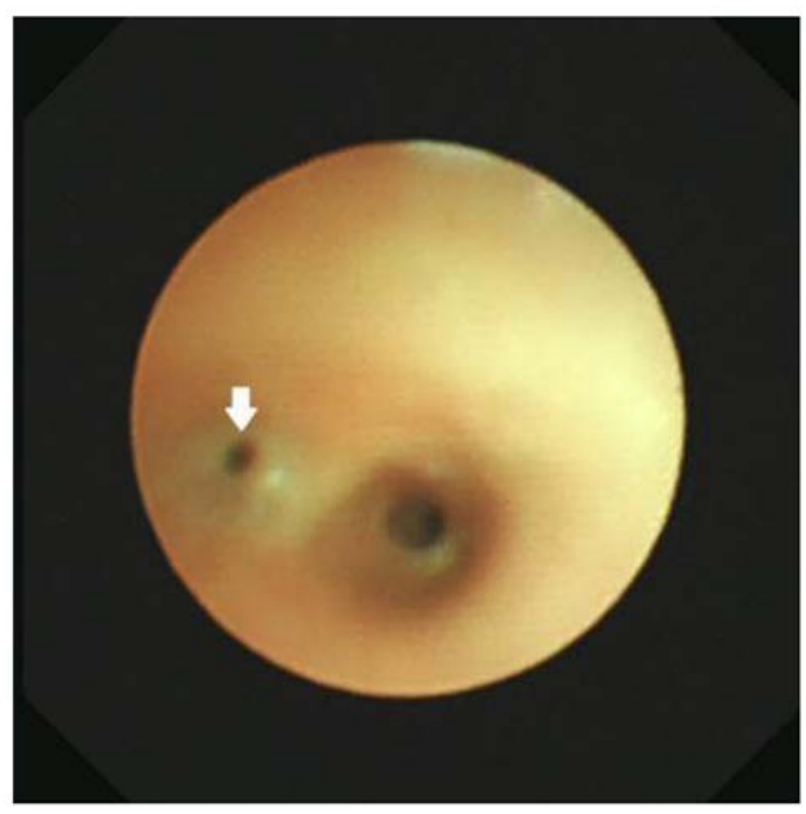

Figure 2. Esophagotracheal fistula. Arrow indicates the fistula.

procedure, it was noticed that in 3 patients the probe of $\mathrm{FB}$ could not pass through the glottis; one case presented severe epiglottis malacia and severe glottic stenosis was detected in 2 cases. There were 27 patients $(50 \%)$ with different degrees of respiratory tract malacia and 18 (33.3\%) exhibited different degrees of respiratory tract stenosis. In addition, 9 cases $(16.7 \%)$ were diagnosed with both malacia and stenosis while 6 cases (11.1\%) had esophagotracheal fistula (Figs. 2-7).

Outcomes. In the experiment group, 44 neonates $(81.4 \%)$ were discharged with improved condition, 5 (9.3\%) succumbed and $5(9.3 \%)$ abandoned the treatment and left the hospital. Among the patients who were discharged, 3 were treated with a tracheostomy and went home with trachea cannula and 1 presented with epiglottic anomaly and the FB could not pass the vocal cord. The other 2 cases presented laryngomalacia combined with trachobronchomalacia. Among the 5 mortalities, 1 was identified with severe glottic stenosis and the FB could not pass, 1 was observed with multiple-malformations of bones, annular stenosis at the middle of trachea, stenosis at the opening of bilateral main bronchi and severe stenosis at left lower bronchus. Severe endobronchitis with tracheal stenosis, mild bronchomalacia with endobronchitis, bronchomalacia combined with stenosis at the left main bronchus were observed in the other 3 cases, respectively. These last 3 cases were all intubated, but succumbed as parents decided to relinquish all

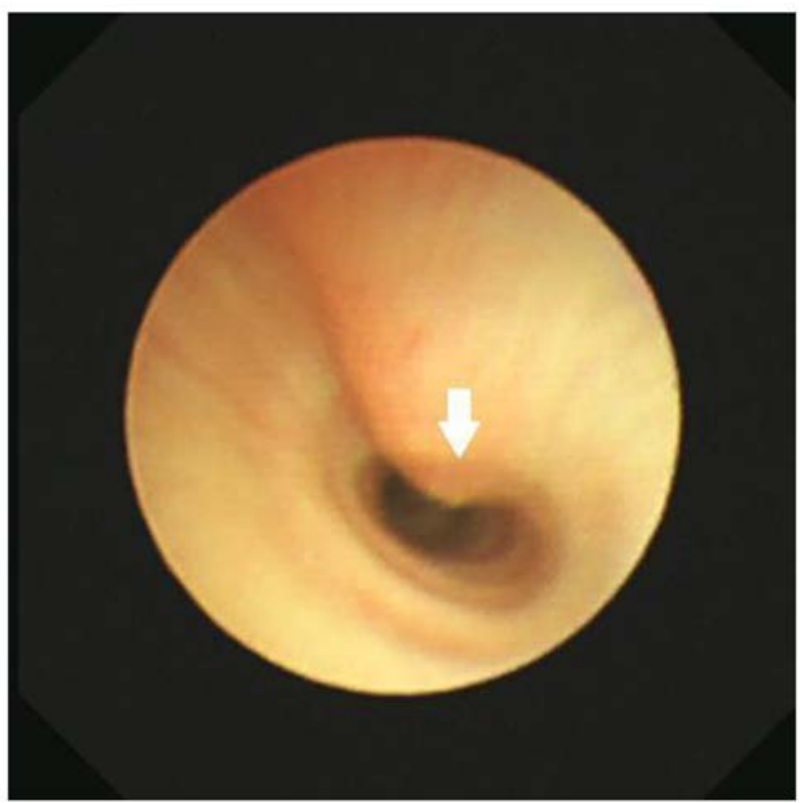

Figure 3. Tracheal stenosis by external pressure. Arrow indicates the respiratory tract stenosis.

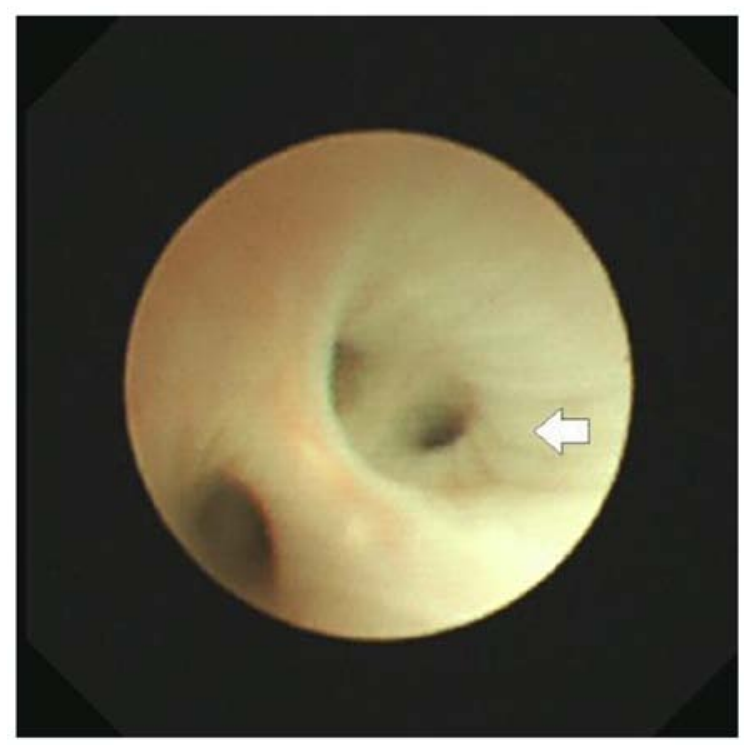

Figure 4. Endobronchitis (pale mucosa with floc). Arrow indicates the manifestation of endobronchitis.

medical treatments. In all, 34 cases were examined by X-ray or CT scan prior to and following FB and improvement on the radiological images was observed in 21 cases $(61.8 \%)$. 


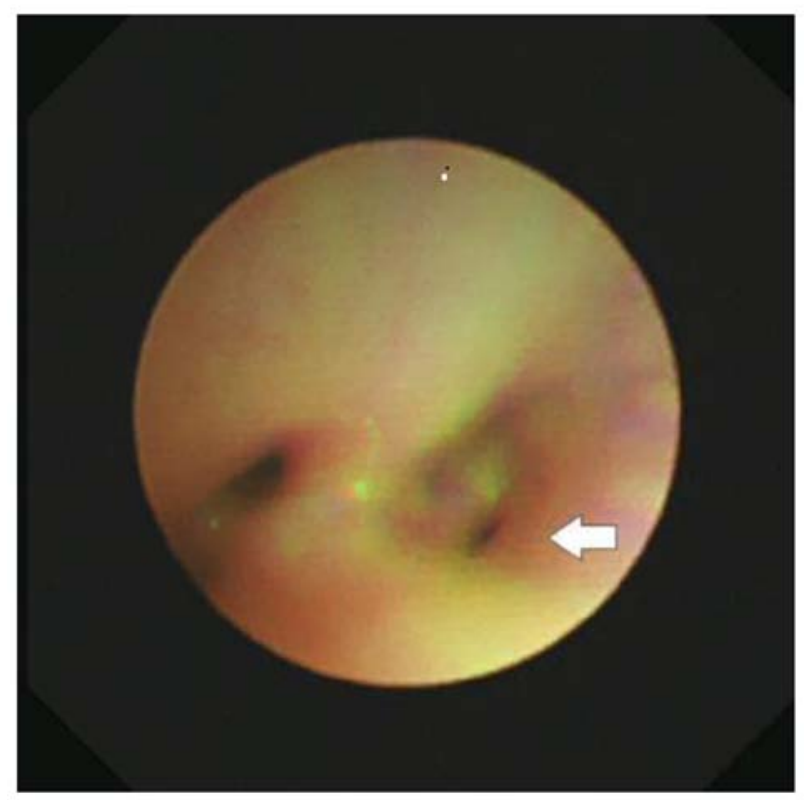

Figure 5. Bronchial stenosis. Arrow indicates the respiratory tract stenosis.

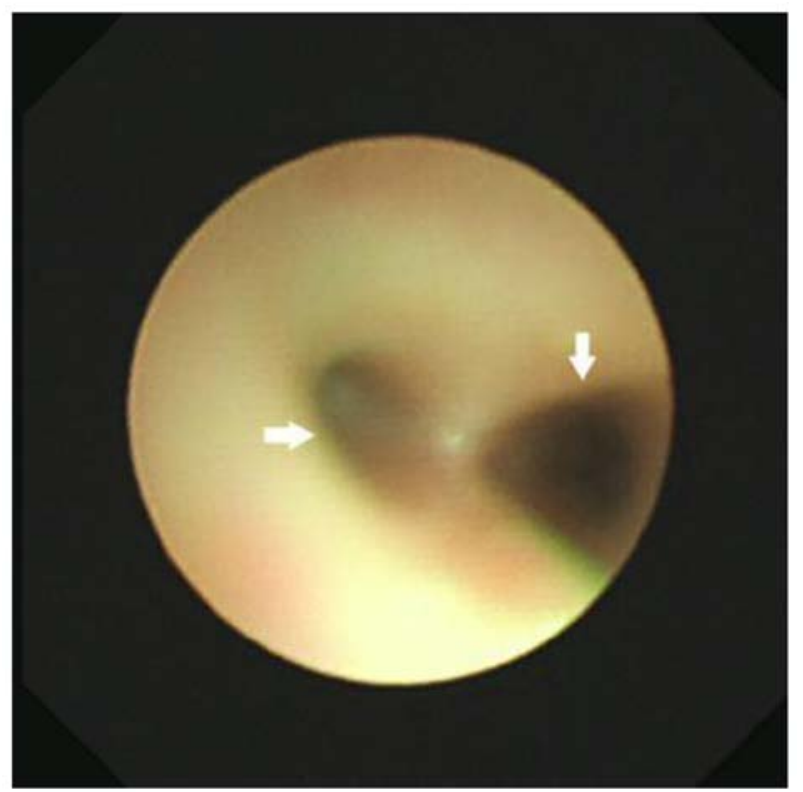

Figure 6. Stenosis at the opening of left main bronchus. Both arrows indicate the respiratory tract stenosis.

$B A L$. Among the 54 patients, 28 received BAL. BAL fluid samples were collected for cell counts, biochemistry and culture test. For the BAL fluid culture, 24 cases tested as positive $(85.7 \%)$ while 4 were negative $(14.3 \%)$. Consistent results between the BAL fluid culture and respiratory secretion culture or tracheal tube culture were identified in 21 cases (75\%; Fig. 8).

Safety of $F B$. Blood gases were detected at $1 \mathrm{~h}$ before and $1 \mathrm{~h}$ after FB for the patients in the experimental group. The results demonstrated that there were no statistical differences in the values of $\mathrm{pH}$, partial pressure of carbon dioxide $\left(\mathrm{PCO}_{2}\right)$ and $\mathrm{HCO}_{3}{ }^{-}$. However, partial pressure of oxygen

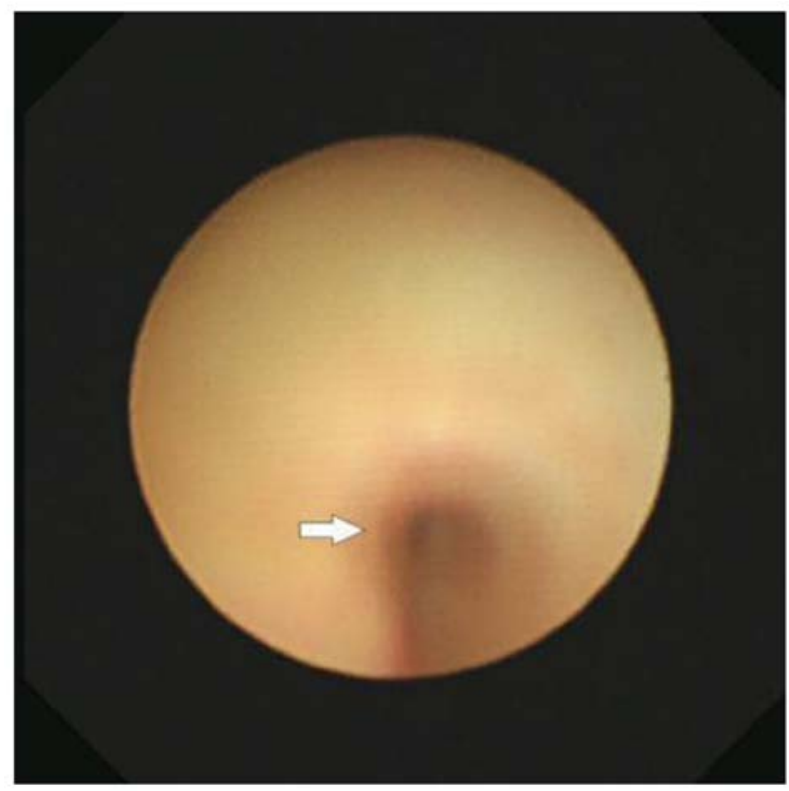

Figure 7. Bronchomalacia. Arrow indicates the severe epiglottis malacia.

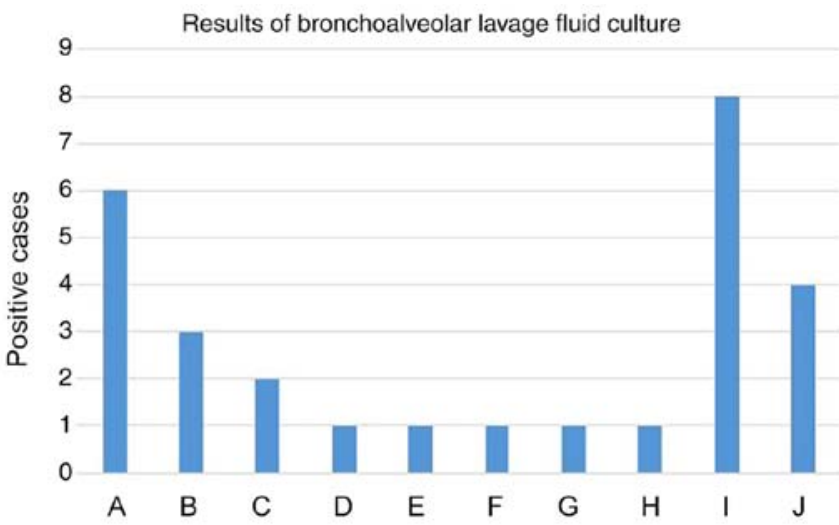

Figure 8. Results of bronchoalveolar lavage fluid culture. A, Acinetobactor baumanii; B, Klebsiella pneumoniae; C, Pseudomonas aeruginosa; D, Klebsiella oxytoca; E, Escherichia coli; F, Staphylococcus haemolyticus; G, Stenotrophomonas maltophilia; $\mathrm{H}$, Enterobacter cloacae; I, Streptococcus viridans and/or Neisseria; J, Negative.

$\left(\mathrm{PO}_{2}\right)$ was significantly higher following $\mathrm{FB}$ procedure compared with that prior to $\mathrm{FB}(\mathrm{P}<0.05$; Table III). Blood gases were also tested at $1 \mathrm{~h}$ before and $1 \mathrm{~h}$ after atomization and secretion suction in the patients of the control group. The results demonstrated that there were no significant differences in $\mathrm{pH}, \mathrm{PCO}_{2}, \mathrm{PO}_{2}$ and $\mathrm{HCO}_{3}^{-}$(Table IV). In comparison between the experimental group and control group, no significant difference in $\mathrm{pH}, \mathrm{PCO}_{2}, \mathrm{PO}_{2}$ and $\mathrm{HCO}_{3}{ }^{-}$was observed (Table $\mathrm{V}$ ). No statistical differences were observed in white blood cell count, hemoglobin, platelet count and neutrophil ratio prior to and following FB. Notably, CRP was significantly decreased following FB $(\mathrm{P}<0.05$; Table VI).

Complications. During the $56 \mathrm{FB}$ procedures performed on 54 patients, $\mathrm{SaO}_{2}$ decreased to $\sim 80 \%$ in 13 cases (23.3\%). After a transient pause, oxygen suction was provided through the 
Table III. Blood gas analysis before and after flexible bronchoscopy.

\begin{tabular}{lccrr}
\hline Blood gas & Before & After & t-test & P-value \\
\hline $\mathrm{pH}$ & $7.39 \pm 0.08$ & $7.40 \pm 0.07$ & -0.627 & 0.535 \\
$\mathrm{PCO}_{2}(\mathrm{mmHg})$ & $49.03 \pm 9.95$ & $47.78 \pm 11.39$ & 0.791 & 0.434 \\
$\mathrm{PO}_{2}(\mathrm{mmHg})$ & $46.83 \pm 13.36$ & $58.32 \pm 24.10$ & -2.688 & 0.011 \\
$\mathrm{HCO}_{3}^{-}(\mathrm{mmol} / \mathrm{l})$ & $28.77 \pm 5.07$ & $29.19 \pm 5.00$ & -0.787 & 0.436 \\
\hline
\end{tabular}

$\mathrm{n}=38, \mathrm{P}<0.05$ was considered to indicate a statistically significant difference. $\mathrm{PCO}_{2}$, partial pressure of carbon dioxide; $\mathrm{PO}_{2}$, partial pressure of oxygen.

Table IV. Blood gas analysis before and after atomization and secretion suction.

\begin{tabular}{lccrr}
\hline Blood gas & Before & After & t-test & P-value \\
\hline $\mathrm{pH}$ & $7.35 \pm 0.10$ & $7.36 \pm 0.09$ & -1.477 & 0.148 \\
$\mathrm{PCO}_{2}(\mathrm{mmHg})$ & $47.93 \pm 12.47$ & $45.65 \pm 10.66$ & 1.253 & 0.218 \\
$\mathrm{PO}_{2}(\mathrm{mmHg})$ & $44.95 \pm 19.88$ & $48.3 \pm 17.22$ & -1.556 & 0.128 \\
$\mathrm{HCO}_{3}^{-}(\mathrm{mmol} / \mathrm{l})$ & $25.70 \pm 3.29$ & $25.63 \pm 3.40$ & 0.175 & 0.862
\end{tabular}

$\mathrm{n}=38, \mathrm{P}<0.05$ was considered to indicate a statistically significant difference. $\mathrm{PCO}_{2}$, partial pressure of carbon dioxide; $\mathrm{PO}_{2}$, partial pressure of oxygen.

Table V. Blood gas analysis comparison between experimental and control groups.

\begin{tabular}{|c|c|c|c|c|}
\hline Blood gas & Experimental group & Control group & t-test & P-value \\
\hline $\mathrm{pH}$ & $-0.01 \pm 0.09$ & $-0.02 \pm 0.07$ & 0.426 & 0.672 \\
\hline $\mathrm{PCO}_{2}(\mathrm{mmHg})$ & $1.25 \pm 9.74$ & $2.28 \pm 11.22$ & -0.380 & 0.706 \\
\hline $\mathrm{PO}_{2}(\mathrm{mmHg})$ & $-11.5 \pm 26.37$ & $-3.35 \pm 13.28$ & -1.707 & 0.096 \\
\hline $\mathrm{HCO}_{3}^{-}(\mathrm{mmol} / \mathrm{l})$ & $-0.43 \pm 3.33$ & $0.07 \pm 2.41$ & -0.756 & 0.455 \\
\hline
\end{tabular}

$\mathrm{n}=38, \mathrm{P}<0.05$ was considered to indicate a statistically significant difference. $\mathrm{PCO}_{2}$, partial pressure of carbon dioxide; $\mathrm{PO}_{2}$, partial pressure of oxygen.

Table VI. Complete blood count and CRP before and after flexible bronchoscopy.

\begin{tabular}{|c|c|c|c|c|}
\hline Complete blood count & Before & After & t-test & P-value \\
\hline White blood cells $\left(10^{9} / 1\right)$ & $14.31 \pm 5.62$ & $13.42 \pm 5.84$ & 1.363 & 0.179 \\
\hline Hemoglobin $(\mathrm{g} / \mathrm{l})$ & $135.28 \pm 29.36$ & $131.44 \pm 32.28$ & 1.397 & 0.169 \\
\hline Platelets $\left(10^{9} / 1\right)$ & $382.94 \pm 170.24$ & $385.42 \pm 158.37$ & -0.187 & 0.852 \\
\hline Neutrophils (\%) & $54.62 \pm 14.15$ & $50.86 \pm 16.57$ & 1.987 & 0.053 \\
\hline $\mathrm{CRP}(\mathrm{mg} / \mathrm{l})$ & $6.06 \pm 10.90$ & $4.04 \pm 6.29$ & 2.335 & 0.024 \\
\hline
\end{tabular}

$\mathrm{n}=50, \mathrm{P}<0.05$ was considered to indicate a statistically significant difference. CRP, C-reactive protein.

operation tunnel and $\mathrm{SaO}_{2}$ increased quickly in all 13 cases. The patients in all 13 cases could tolerate the intervention until FB was completed. Mild tracheal or bronchial mucosa hemorrhage occurred in 13 cases $(23.3 \%)$, but no case presented severe bleeding. No pneumothorax, shock or other severe complications, which might impede the procedure were identified. No fever or diffused pneumonia was observed following FB. 


\section{Discussion}

Flexible bronchoscopy (FB) has developed rapidly in pediatrics in recent years (7). In addition to the value of observation and diagnosis as endoscopy, FB can be used to remove foreign bodies, provide guidance for bronchoalveolar lavage (BAL), intubation and balloon dilatation surgery and as a tool for treatments $(5,6)$. At present, there are four different models of bronchoscope in pediatrics. The inner diameters are 4.9, 3.5, 2.8 and $2.2 \mathrm{~mm}$, respectively, with optional operation tunnels. The smallest one is the $2.2-\mathrm{mm}$ diameter probe with no operation tunnel, which can only be used to observe and diagnose. The $2.8-\mathrm{mm}$ diameter probe is widely used in neonatal wards, because it can be operated through a 3.5 or $4 \mathrm{~mm}$ tracheal tube and can be accompanied with an operation tunnel for oxygen supply. Continuous or recurrent dyspnea and stridor frequently occur in some patients in NICU. Occasionally the confirmatory diagnosis cannot be made by regular radiological examination. For these patients, FB presents an alternative to detect anomalies of the respiratory tract. He et al (8) reported that pathological changes were identified in 73 cases out of 82 patients $(89.0 \%)$, in which 24 cases were diagnosed with congenital respiratory tract malformations (39.3\%). In the present study, respiratory construction problems were identified in 44 cases out of 54 patients (81.5\%). In NICU, due to different reasons, many clinically indicated neonates cannot receive FB examination: It may lead to misdiagnosis and affect the treatment outcomes, resulting in a high positive rate of anomalies.

Respiratory tract malacia, including laryngomalacia, tracheomalacia and bronchomalacia, represents the most common respiratory malformation (9). In neonates and children with stridor, the morbidity of laryngonalacia is $60-70 \%$ (10). The majority of patients with laryngomalacia do not require intervention. However, some severe cases can progress to pulmonary hypertension and pulmonary heart disease due to a lack of appropriate treatments. As laryngonalacia is poorly tolerated in $10 \%$ of cases, assessment and surgical management as well as management of any associated gastro-esophageal reflux are often required to effectively control symptoms (11). For instance, $5-20 \%$ of children with severe or refractory disease may require a more aggressive intervention, most commonly in the form of transoral supraglottoplasty (12). Erdem et al (13) reported that in 109 infants with stridor, 37 cases were identified with isolated laryngomalacia, 54 cases with laryngomalacia with secondary airway lesions, including tracheomalacia, bronchomalacia and tracheobronchomalacia. Only 19 patients received surgery, of which 12 had a tracheostomy. The present study identified 14 patients with laryngomalacia, of which three received tracheostomy. However, Olney et al (14) recommended that FB should not be routinely performed in laryngomalacia; only if there is evidence of concomitant airway lesion. The symptoms of tracheobronchomalacia are not distinctive, but it is the most common cause of recurrent stridor and cough in infants. According to its etiology, tracheomalacia can be divided into two types: Primary tracheomalacia and secondary tracheomalacia. Primary tracheomalacia is a congenital condition. It may represent an isolated finding or be associated with other congenital anomalies, such as cleft palate, choanal atresia and esophageal atresia. Secondary tracheomalacia is always caused by trauma, extratracheal compression, positive pressure ventilation, respiratory tract infection or inflammation (15). In preterms with bronchopulmonary dysplasia, secondary tracheomalacia is very common (16). The present study identified 19 cases of tracheomalacia, bronchomalacia or tracheobronchomalacia out of 54 patients (35.2\%). Neonates with respiratory tract malacia will progress to obstruction with secretion following airway infection. Malacia can lead to dyspnea, atelectasis, recurrent respiratory infection and difficulty in extubation. Therefore, the positive rate of respiratory tract malacia is high in the indicated neonates of FB. The majority of these patients require only supportive treatments, but some, who have severe symptoms of obstruction, may require surgery (17-19). Congenital heart disease is the main reason for secondary tracheomalacia and tracheal stenosis. Lee et al (20) reported that in the children with congenital heart disease and respiratory obstructive problems, $67 \%$ of them were diagnosed by FB with tracheal stenosis due to extratracheal compression. The present study identified two cases with tracheal stenosis caused by extratracheal compression associated with a large ventricular septal defect. FB is also important to surgical departments. It offers useful assistance for thoracic, otolarynological and some general surgeries (21-24).

BAL can remove the secretion in the respiratory tract to remove obstructions. BAL fluid culture provides confirmatory identification of the specific pathogen and guides the anti-infection treatment. The testing of BAL fluid is an important method to assist clinic diagnosis and predict outcomes (25-27). Currently the research on BAL has moved from the cellular to the molecular level (28-30).

There remains a safety concern about FB in neonatal units and complications of FB are frequently identified, including laryngeal edema, laryngeal spasm, hemorrhage, pneumothorax or mediastinal emphysema, hypoxia and fever (31). However, in pediatric clinical practice, severe complications rarely happen (32). It is even safe for patients who receive extracorporeal membrane oxygenation treatment (33). Soong et al (34) reported that the oxygen supplied by using nasopharyngeal catheter during FB is a simple and cost-effective method to maintain appropriate $\mathrm{SaO}_{2}$. In line with the previous findings, the present study illustrated the favorable improvements due to FB in neonates, although there were a number of side effects during the procedure. Overall, 44 neonates $(81.4 \%)$ were discharged with an improved condition, 5 cases $(9.3 \%)$ succumbed and 5 patients $(9.3 \%)$ abandoned the treatment and left the hospital. Overall, no significant difference in terms of $\mathrm{pH}, \mathrm{PCO}_{2}, \mathrm{PO}_{2}$ and $\mathrm{HCO}_{3}{ }^{-}$ was identified between the experimental and control groups. However, $\mathrm{PO}_{2}$ was significantly increased, whereas CRP was significantly reduced following FB procedure compared with prior to FB $(\mathrm{P}<0.05)$. No fever or diffused pneumonia was observed following FB. A limitation in the present study was that the clinical safety requires further validation with a larger number of patients in NICU. As for the sedation during the FB procedure, midazolam in combination with 
fentanyl or alfentanil is the first choice (28). More studies are required to examine the best way to decrease damage caused by $\mathrm{FB}$ in neonates.

In conclusion, $\mathrm{FB}$ possesses important value in diagnosis and differentiation in neonatal respiratory diseases. BAL can be a useful treatment in atelectasis. FB is relatively safe in clinical practice in NICU and severe complications rarely occur.

\section{Acknowledgements}

The authors would like to thank Dr Lu Min, Dr Shu Linhua and Dr Gu Haoxiang of the Respiratory Department, Shanghai Children's Hospital, Shanghai, China.

\section{Funding}

No funding was received.

\section{Availability of data and materials}

The datasets used and/or analyzed during the current study are available from the corresponding author on reasonable request.

\section{Authors' contributions}

CY and YH contributed to the conception and design of the study and performed the experiments. GQ, XG and DE contributed to acquisition, analysis and interpretation of data. CY drafted the manuscript and revised it critically for important intellectual content and gave final approval of the version to be published. All authors read and approved the final manuscript.

\section{Ethics approval and consent to participate}

The present study was reviewed and approved by the Ethics Committee of Shanghai Children's Hospital, Shanghai, China (approval no. 2011-231). All families participated in the present study voluntarily and signed informed consent.

\section{Patient consent for publication}

Not applicable.

\section{Competing interests}

The authors declare that they have no competing interests.

\section{References}

1. Pereira W, Kovnat DM, Khan MA, Iacovino JR, Spivack ML and Snider GL: Fever and pneumonia after flexible fiberoptic bronchoscopy. Am Rev Respir Dis 112: 59-64, 1975.

2. Zhang W, Yang YX, Yu W and Qi SH: Cough Suppression during flexible bronchoscopy using transcutaneous electric acupoint stimulation: A randomized controlled study. Evid Based Complement Alternat Med 2019: 5650413, 2019.

3. Ndilanha DA, Shayo GA, Hassan R, Byomuganyizi M and Lema LEK: Diagnoses from lung specimen collected through flexible bronchoscopy from patients in a tertiary hospital in Dar es Salaam Tanzania: A retrospective cross-sectional study. BMC Pulm Med 19: 214, 2019.
4. Sachdev A, Chhawchharia R, Gupta D and Gupta N: Flexible fiberoptic bronchoscopy directed interventions in neonatal intensive care unit. Indian Pediatr 56: 563-565, 2019.

5. The Pediatric Bronchoscopy Collaborative Group, the Subspecialty Group of Respiratory Diseases, the Society of Pediatrics, Chinese Medical Association. Guide to pediatric bronchoscopy (2009 edition). Chin J Pediatr 47: 740-744, 2009.

6. Pérez-Frías J, Moreno Galdó A, Pérez Ruiz E, Barrio Gómez De Agüero MI, Escribano Montaner A and Caro Aguilera P; Sociedad Española de Neumología y Cirugía Torácica: Pediatric bronchoscopy guidelines. Arch Bronconeumol 47: 350-360, 2011 (In Spanish).

7. Hysinger EB, Hart CK, Burg G, De Alarcon A and Benscoter D: Differences in flexible and rigid bronchoscopy for assessment of tracheomalacia. Laryngoscope: Apr 13, 2020 (Epub ahead of print). doi: 10.1002/lary.28656.

8. He SR, Sun YX, Yu YH, Liu YM, Zhong J, Liang SX, et al: The applications of flexible fiberoptic bronchoscopy in NICU. J Clin Pediatr 27: 18-21, 2009.

9. Pan W, Peng D, Luo J, Liu E, Luo Z, Dai J, Fu Z, Li Q and Huang Y: Clinical features of airway malacia in children: A retrospective analysis of 459 patients. Int J Clin Exp Med 7: 3005-3012, 2014

10. Daniel SJ: The upper airway: Congenital malformations. Paediatr Respir Rev 7 (Suppl 1): S260-S263, 2006.

11. Ayari S, Aubertin G, Girschig H, Van Den Abbeele T, Denoyelle F, Couloignier V and Mondain M: Management of laryngomalacia. Eur Ann Otorhinolaryngol Head Neck Dis 130: 15-21, 2013.

12. Thorne MC and Garetz SL: Laryngomalacia: Review and summary of current clinical practice in 2015. Paediatr. Respir Rev 17: 3-8, 2016.

13. Erdem E, Gokdemir Y, Unal E, Ersu R, Karadag B and Karakoc F: Flexible bronchoscopy as a valuable tool in the evaluation of infants with stridor. Eur Arch Otorhinolaryngol 270: 21-25, 2013.

14. Olney DR, Greinwald JH Jr, Smith RJ and Bauman NM: Laryngomalacia and its treatment. Laryngoscope 109: 1770-1775, 1999.

15. Majid A, Fernandez L, Bussy SF, Herth F and Ernst A: Tracheobronchomalacia. Arch Bronconeumol 46: 196-202, 2010 (In Spanish).

16. Hysinger EB and Panitch HB: Paediatric Tracheomalacia. Paediatr Respir Rev 17: 9-15, 2016.

17. Wright CD: Treatment of congenital tracheal stenosis. Thorac Cardiovasc Surg 21: 274-277, 2009.

18. Jaquiss RD: Management of pediatric tracheal stenosis and tracheomalacia. Semin Thorac Cardiovasc Surg 16: 220-224, 2004

19. Fraga JC, Jennings RW and Kim PC: Pediatric tracheomalacia. Semin Pediatr Surg 25: 156-164, 2016.

20. Lee S, Cheung YF, Leung MP, Ng YK and Tsoi NS: Airway obstruction in children with congenital heart disease: Assessment by flexible bronchoscopy. Pediatr Pulmonol 34: 304-311, 2002.

21. Yang ZY, Liang L, Liu WJ, Zhang Q, Jin DQ, Jiang R, et al: Application of fiberoptic bronchoscopy on diagnosis and treatment of respiratory comorbidity in children with congenital heart disease. J Appl Clin Pediatr 26: 260-261, 2011.

22. Sun YX, He SR, Liang SX, Zhong J, Liu YM, Ge PJ, et al: Dilation guided by fiberoptic bronchoscope treating for subglottic stenosis in infants. J Appl Clin Pediatr 25: 1758-1761, 2010.

23. Atzori P, Lacobelli BD, Bottero S, Spirydakis J, Laviani R, Trucchi A, Braguglia A and Bagolan P: Preoperative tracheobronchoscopy in newborns with esophageal atresia: Does it matter? J Pediatr Surg 41: 1054-1057, 2006.

24. Parolini F, Boroni G, Stefini S, Agapiti C, Bazzana T and Alberti D: Role of preoperative tracheobronchoscopy in newborns with esophageal atresia: A review. World J Gastrointest Endosc 6: 482-487, 2014.

25. Antabak A, Luetic T, Caleta D and Romic I: H-type tracheoesophageal fistula in a newborn: Determining the exact position of fistula by intra-operative guidewire placement. J Neonatal Surg 3: 36, 2014.

26. Finke MD: Transtracheal wash and bronchoalveolar lavage. Top Companion Anim Med 28: 97-102, 2013.

27. Torre $\mathrm{O}$ and Harari S: The diagnosis of cystic lung diseases: A role for bronchoalveolar lavage and transbronchial biopsy? Respir Med 104 (Suppl 1): S81-S85, 2010.

28. Palomares LJ, Juan JM, Izquierdo LG, Dominguez AC, Becerra ER and Panadero FR: Bronchoalveolar lavage findings in patients with diffuse interstitial lung disease: Prospective study of a cohort of 562 patients. Arch Bronconeumol 45: 115-121, 2009. 
29. Noël-Georis I, Bernard A, Falmagne P and Wattiez R: Database of bronchoalveolar lavage fluid proteins. J Chromatogr B Analyt Technol Biomed Life Sci 771: 221-236, 2002.

30. Wattiez R and Falmagne P: Proteomics of bronchoalveolar lavage fluid. J Chromatogr B Analyt Technol Biomed Life Sci 815: 169-178, 2005.

31. Tang LF, Xu YC, Wang YS, Wang CF, Zhu GH, Bao XE, Lu MP, Chen LX and Chen ZM: Airway foreign body removal by flexible bronchoscopy: Experience with 1027 children during 2000-2008. World J Pediatr 5: 191-195, 2009

32. Chen WM, Lu GP, Lu ZJ and Zhang LE: The application of bronchofibroscope in PICU. Chin Pediatr Emergency Med 18: 129-132, 2011.
33. Kamat PP, Popler J, Davis J, Leong T, Piland SC, Simon D, Harsch A, Teague WG and Fortenberry JD: Use of flexible bronchoscopy in pediatric patients receiving extracorporeal membrane oxygenation (ECMO) Support. Pediatr Pulmonol 46: 1108-1113, 2011.

34. Soong WJ, Lee YS, Tsao PC, Yang CF and Jeng MJ: Comparison of oxygenation among different supplemental oxygen methods during flexible bronchoscopy in infants. J Chin Med Assoc 74: 556-560, 2011. 